\title{
IMPLEMENTAÇÃO E USO DIÁRIO DA LISTA DE VERIFICAÇÃO DE SEGURANÇA CIRÚRGICA EM HOSPITAIS
}

\author{
Implementation and daily use of the surgical safety checklist in hospitals \\ Implementación y uso diario de la lista de control de seguridad quirúrgica en hospitales \\ Maria Fernanda do Prado Tostes ${ }^{1}$ (1), Cristina Maria Galvão ${ }^{2}$ (1)
}

RESUMO: Objetivo: Identificar o processo de implementação e o uso diário da lista de verificação de segurança cirúrgica, segundo relato de enfermeiros que atuavam no centro cirúrgico de hospitais. Método: Estudo descritivo-exploratório, com amostra de 77 enfermeiros. A coleta de dados ocorreu durante seis meses, no ano de 2016, por meio da aplicação de instrumento elaborado e submetido à validação de face e conteúdo. Na análise dos dados, adotou-se a estatística descritiva. Resultados: A realização de programa educacional foi essencial no processo de implementação da lista de verificação de segurança cirúrgica. O circulante era o responsável pela checagem diária da ferramenta na sala cirúrgica. A maioria dos enfermeiros relatou adesão parcial no uso do checklist pela equipe cirúrgica; houve diferença de adesão entre as etapas de checagem (entrada, pausa e saída) e entre as categorias profissionais. Conclusão: O conhecimento produzido oferece subsídios para a implementação da ferramenta e o uso de estratégias que podem auxiliar no cotidiano da prática clínica. Palavras-chave: Lista de checagem. Organização Mundial da Saúde. Segurança do paciente. Enfermagem perioperatória.

ABSTRACT: Objective: To identify the implementation process and the daily use of the surgical safety checklist, according to the report of nurses who worked in the surgical center of hospitals. Method: Descriptive-exploratory study, with a sample of 77 nurses. Data collection took place over six months in 2016, with the application of an instrument prepared and submitted to face and content validation. In the data analysis, descriptive statistics was adopted. Results: The realization of an educational program was essential in the process of implementing the surgical safety checklist. The circulator nurse was responsible for checking the instrument daily in the operating room. Most nurses reported partial adherence to the use of the checklist by the surgical team; there was difference in adherence between the checking stages (sign in, time out, and sign out) and between professional categories. Conclusion: The knowledge produced offers subsidies for the implementation of the instrument and the use of strategies that can assist in daily clinical practice. Keywords: Checklist. World Health Organization. Patient safety. Perioperative nursing.

RESUMEN: Objetivo: Identificar el proceso de implementación y uso diario de la lista de verificación de seguridad quirúrgica, según el informe de enfermeras que laboraron en el centro quirúrgico de los hospitales. Método: Estudio descriptivo-exploratorio, con una muestra de 77 enfermeros. La recolección de datos se llevó a cabo durante seis meses, en 2016, mediante la aplicación de un instrumento elaborado y sometido a validación facial y de contenido. En el análisis de los datos se adoptó estadística descriptiva. Resultados: La realización de un programa educativo fue fundamental en el proceso de implementación de la lista de verificación de seguridad quirúrgica. El circulador se encargaba de revisar la herramienta diariamente en el quirófano. La mayoría de las enfermeras informaron un cumplimiento parcial del uso de la lista de verificación por parte del equipo quirúrgico; hubo una diferencia en la adherencia entre los pasos de verificación (entrada, pausa y salida) y entre categorías profesionales. Conclusión: El conocimiento producido ofrece subsidios para la implementación de la herramienta y el uso de estrategias que pueden ayudar en la práctica clínica diaria.

Palabras clave: Lista de verificación. Organización Mundial de la Salud. Seguridad del paciente. Enfermería perioperatoria.

'Enfermeira. Doutora em Ciências pela Escola de Enfermagem de Ribeirão Preto da Universidade de São Paulo. Docente do Colegiado de Enfermagem da Universidade Estadual do Paraná - Paranavaí (PR), Brasil. ${ }^{2}$ Enfermeira. Pós-Doutora em Enfermagem pela Universidade de Alberta. Professora titular da Escola de Enfermagem de Ribeirão Preto da Universidade de São Paulo - São Paulo (SP), Brasil.

*Autora correspondente: mfpprado@gmail.com

Recebido: 13/05/2020. Aprovado: 09/10/2020

https://doi.org/10.5327/Z1414-4425202000040003 


\section{INTRODUÇÃO}

Desde 2008, a Lista de Verificação de Segurança Cirúrgica (LVSC), desenvolvida pela Organização Mundial da Saúde (OMS), é recomendada para melhorar a segurança do paciente cirúrgico ${ }^{1}$. Após 12 anos da criação dessa ferramenta, o trabalho em equipe, a comunicação e a segurança do paciente foram aspectos que melhoraram com o seu uso nos hospitais ${ }^{2}$.

No contexto mundial, por meio de estudos, divulgaram-se as experiências com a implementação da LVSC, e os resultados obtidos indicaram que a ferramenta foi adaptada e implementada de maneira distinta entre os serviços de saúde, havendo a necessidade de integrar diferentes estratégias para melhorar as práticas de segurança cirúrgica e alcançar os benefícios do uso do checklist em cenários diversos ${ }^{3,4}$.

Nos hospitais, a implementação da LVSC consiste em processo complexo e desafiador, pois requer que equipes cirúrgicas mudem comportamentos e aprendam novos hábitos ${ }^{5,6}$. Ademais, estudiosos da problemática afirmam que, muitas vezes, a forma como a ferramenta foi posta em prática acarretou execução incompleta ou inconsistente ${ }^{4}$.

No Brasil, as evidências científicas disponíveis abrangeram conhecimento, percepção e atitudes da equipe, adesão e impacto da $\mathrm{LVSC}^{7,8}$. Contudo os estudos desenvolvidos pouco exploraram o processo de implementação do checklist $^{6-9}$.

Ademais, em revisão de literatura, os autores destacaram que, nos países em desenvolvimento, como o Brasil, existe escassez de estudos em relação a todos os aspectos do uso da LVSC que precisam ser abordados. Para defender continuamente a LVSC como medida de segurança cirúrgica aplicável à população global, a pesquisa nesses países precisa ser prioridade. As estratégias de implementação devem ser investigadas no contexto dos países em desenvolvimento para que os hospitais possam avaliar a abordagem mais adequada para introduzir o uso do checklist e mais compatível com a realidade local, a fim de promover o incremento da utilização dessa ferramenta e beneficiar mais pacientes ${ }^{9}$.

Considerando o potencial da LVSC para melhorar a segurança do paciente cirúrgico e a necessidade de gerar evidências que possam contribuir com a redução de lacunas do conhecimento, a condução do presente estudo pautou-se na seguinte questão de pesquisa: como ocorreu o processo de implementação e o uso diário da LVSC nos hospitais investigados?

\section{OBJETIVO}

Identificar o processo de implementação e o uso diário da lista de verificação de segurança cirúrgica, segundo relato de enfermeiros que atuavam no centro cirúrgico de hospitais.

\section{MÉTODO}

Trata-se de estudo descritivo-exploratório, realizado em 16 hospitais que implementaram a LVSC, de duas principais cidades paranaenses, sendo $11 \mathrm{em}$ Londrina e $5 \mathrm{em}$ Maringá. Para a seleção dos hospitais, o Cadastro Nacional de Estabelecimentos de Saúde (CNES) do Ministério da Saúde (MS) foi consultado.

A população-alvo foram os enfermeiros com atuação na unidade de centro cirúrgico (CC) dos hospitais selecionados, a saber: enfermeiro coordenador ou enfermeiro assistencial. Os enfermeiros sem atuação exclusiva no setor foram excluídos, além dos profissionais que estivessem cobrindo folga ou férias ou em licença.

A população era de 81 enfermeiros atuantes em CC, sendo 58 nos hospitais de Londrina e 23 em Maringá. Do total, após a aplicação dos critérios de seleção, quatro enfermeiros foram excluídos: dois profissionais não concordaram em participar da pesquisa, um estava de atestado médico e um de licença maternidade. Assim, a amostra foi composta de 77 enfermeiros, sendo 56 em Londrina e 21 em Maringá.

Utilizou-se um instrumento elaborado pelas pesquisadoras para a coleta de dados, o qual foi submetido à validade de face e de conteúdo por três enfermeiros (juízes), com atividades de ensino e/ou pesquisa em enfermagem perioperatória. $\mathrm{O}$ instrumento incluiu dados sobre a caracterização dos enfermeiros, do hospital e do CC e dados sobre a implementação e o uso diário da LVSC. A coleta de dados ocorreu durante seis meses, no ano de 2016.

Elaborou-se uma planilha eletrônica no Microsoft Excel para o armazenamento dos dados, e a técnica de dupla digitação foi adotada. O software Statistical Package Social Sciences (SPSS) versão 19.0 foi empregado para a análise dos dados. Descreveram-se as variáveis investigadas pela frequência absoluta $\left(\mathrm{n}^{\circ}\right)$ e relativa (\%), média aritmética e desvio-padrão.

O estudo foi aprovado pelo Comitê de Ética mediante parecer $n^{\circ} 164 / 2015$, CAAE $n^{\circ} 48347115.9 .0000 .5393$, e a participação dos enfermeiros ocorreu por meio da leitura e assinatura do Termo de Consentimento Livre e Esclarecido. 


\section{RESULTADOS}

Dos 77 enfermeiros, a maioria $(72 ; 93,5 \%)$ era do sexo biológico feminino, a média de idade foi de 34,6 anos (desvio padrão $=9,5)$, e o tempo de atuação no CC foi de 5,6 anos (desvio padrão=6,2). A maioria $(46 ; 59,7 \%)$ dos participantes trabalhava em instituição hospitalar privada. A média de tempo de implementação do checklist foi de 27,8 meses. A Tabela 1 apresenta os dados sobre o processo de implementação da LVSC nos hospitais selecionados.

Quanto ao formato da LVSC, "dois ou mais formatos" da ferramenta disponíveis em sala operatória (SO) foi o percentual maior $(35 ; 45,4 \%)$, abrangendo os formatos impresso e pôster instalado na parede da $\mathrm{SO}(13 ; 16,9 \%)$; impresso e eletrônico (13; 16,9\%); eletrônico e pôster (3; 3,9\%) e impresso, eletrônico e pôster (6;7,8\%).

Para a implementação da LVSC nos hospitais, a maioria dos participantes $(65 ; 84,4 \%)$ respondeu que houve realização de programa educacional, oferecido exclusivamente para a equipe de enfermagem.

No que se refere à definição de responsabilidade pela condução da checagem diária da LVSC em SO, o circulante foi a pessoa que recebeu o percentual maior (59; 76,6\%) (Tabela 1). Ressalta-se que, no instrumento de coleta de dados, os respondentes poderiam assinalar uma ou mais opções.

Com relação à iniciativa para a implementação da LVSC nos hospitais, os participantes atribuíram os percentuais maiores aos enfermeiros, a saber: $49(63,6 \%)$ ao enfermeiro do CC, $48(62,3 \%)$ ao enfermeiro coordenador do CC e $38(49,4 \%)$ ao gerente de enfermagem. Dos 77 enfermeiros, $26(33,8 \%)$ atribuíram a iniciativa ao anestesista ou chefe do serviço de anestesia, $14(18,2 \%)$ ao diretor administrativo e $12(15,6 \%)$ ao cirurgião ou chefe do serviço de cirurgia. Salienta-se que, no instrumento de coleta de dados, os enfermeiros poderiam assinalar uma ou mais opções (dados não demonstrados em tabelas).

Com relação ao planejamento, os participantes atribuíram os percentuais maiores aos enfermeiros, na seguinte distribuição: 55 (71,4\%) enfermeiro do CC, 44 (57,1\%) enfermeiro coordenador do CC e $33(42,9 \%)$ gerente de enfermagem. A seguir, o anestesista ou chefe do serviço de anestesia $(25 ; 32,5 \%)$, o cirurgião ou chefe do serviço de cirurgia (12; $15,6 \%)$, o diretor administrativo $(6 ; 7,8 \%)$ e outros profissionais $(9 ; 11,7 \%)$. Ressalta-se que, no instrumento de coleta de dados, os respondentes poderiam assinalar uma ou mais opções (dados não demonstrados em tabelas).

Sobre o uso diário da LVSC pela equipe cirúrgica, para a maioria dos enfermeiros (52;67,5\%), a equipe cirúrgica adere parcialmente ao uso dessa ferramenta. E, para a maioria dos participantes $(50 ; 64,9 \%)$, houve incremento na adesão ao uso do checklist pela equipe cirúrgica, desde a sua introdução no serviço de saúde (Tabela 2).

Na opinião dos enfermeiros ( $n=44), 31(70,5 \%)$ apontaram que a entrada era a etapa que a equipe cirúrgica apresentava maior adesão, e 16 enfermeiros $(36,4 \%)$ indicaram a saída como a etapa com menor adesão (dados não demonstrados na Tabela 2).

Para 64 enfermeiros (83,1\%), existia diferença na adesão ao uso da LVSC entre as categorias profissionais (Tabela 2), e a equipe de enfermagem $(48 ; 75,0 \%)$ tinha maior adesão; para apenas um participante $(1,6 \%)$ o anestesista era a categoria com maior adesão. Com relação aos profissionais com menor adesão ao uso diário do checklist, 35 enfermeiros (54,7\%) relataram os cirurgiões, e $11(17,2 \%)$, os cirurgiões e anestesistas (dados não demonstrados na Tabela 2).

A maioria dos participantes relatou que a checagem da LVSC ocorre verbalmente $(56 ; 72,7 \%)$, contudo apenas 27 $(35,1 \%)$ afirmaram que a equipe cirúrgica completa está presente, presta atenção e participa da checagem (Tabela 2).

A maioria dos enfermeiros $(57 ; 74,0 \%)$ respondeu que todos os itens da LVSC eram checados em SO, mas

Tabela 1. Caracterização do processo de implementação da lista de verificação de segurança cirúrgica nos hospitais, segundo relato dos enfermeiros.

\begin{tabular}{|c|c|c|}
\hline \multirow{2}{*}{ Variáveis } & \multicolumn{2}{|c|}{ Enfermeiros } \\
\hline & $n=77$ & Porcentagem (\%) \\
\hline $\begin{array}{l}\text { Tempo de implementação } \\
\text { (meses) }\end{array}$ & $27,8(21,4)^{*}$ & - \\
\hline \multicolumn{3}{|l|}{ Formato da LVSC } \\
\hline Impresso & 34 & 44,2 \\
\hline Eletrônico & 06 & 7,8 \\
\hline $\begin{array}{l}\text { Quadro fixado na sala } \\
\text { cirúrgica }\end{array}$ & 02 & 2,6 \\
\hline Dois ou mais formatos & 35 & 45,5 \\
\hline \multicolumn{3}{|c|}{ Programa educacional para a equipe cirúrgica } \\
\hline Sim & 65 & 84,4 \\
\hline Não & 12 & 15,6 \\
\hline \multicolumn{3}{|l|}{ Responsável pela checagem } \\
\hline Cirurgião & 3 & 3,9 \\
\hline Enfermeiro coordenador & 18 & 23,4 \\
\hline Anestesista & 11 & 14,3 \\
\hline $\begin{array}{l}\text { Enfermeiro do centro } \\
\text { cirúrgico }\end{array}$ & 43 & 55,8 \\
\hline Circulante & 59 & 76,6 \\
\hline Outros & 15 & 19,5 \\
\hline
\end{tabular}


$19(24,7 \%)$ indicaram a existência de itens que não eram checados (Tabela 2).

Com relação aos itens cuja checagem era negligenciada $(n=19)$, quatro participantes $(21,0 \%)$ apontaram os itens pertencentes à entrada, entre os quais: marcação de lateralidade ( $\mathrm{n}=1)$, investigação sobre o uso de medicamentos e cirurgia prévia $(n=2)$ e via aérea difícil $(n=1)$. Para cinco participantes (26,3\%), foram os itens pertencentes à pausa, a saber: duração estimada da cirurgia $(n=1)$, antecipação de risco adicional $(\mathrm{n}=1)$, todos os itens pertencentes à pausa $(\mathrm{n}=2)$ e questões/preocupações com equipamentos $(\mathrm{n}=1)$. Também cinco enfermeiros $(26,3 \%)$ relataram que foram os itens pertencentes à saída: todos indicaram a contagem cirúrgica e um $(5,3 \%)$ ressaltou os itens que estavam duplicados na LVSC ou que não se aplicavam a determinada cirurgia. Dois participantes $(10,5 \%)$ apontaram os itens

Tabela 2. Caracterização da adesão ao uso diário da lista de verificação de segurança cirúrgica nos hospitais, segundo relato dos enfermeiros.

\begin{tabular}{|c|c|c|}
\hline \multirow{2}{*}{ Variáveis } & \multicolumn{2}{|c|}{ Enfermeiros } \\
\hline & $n=77$ & Porcentagem (\%) \\
\hline \multicolumn{3}{|c|}{ Adesão da equipe cirúrgica ao uso da LVSC } \\
\hline Total & 21 & 27,3 \\
\hline Parcial & 52 & 67,5 \\
\hline Não & 04 & 5,2 \\
\hline \multicolumn{3}{|c|}{ Aumento na adesão desde a introdução da LVSC } \\
\hline Sim & 50 & 64,9 \\
\hline Não & 26 & 33,8 \\
\hline Não respondeu & 01 & 1,3 \\
\hline \multicolumn{3}{|c|}{ Diferença na adesão entre as etapas de checagem } \\
\hline Sim & 44 & 57,1 \\
\hline Não & 33 & 42,9 \\
\hline \multicolumn{3}{|c|}{ Diferença na adesão à LVSC entre profissionais } \\
\hline Sim & 64 & 83,1 \\
\hline Não & 11 & 14,3 \\
\hline Não respondeu & 02 & 2,6 \\
\hline \multicolumn{3}{|c|}{ Checagem verbal da LVSC pela equipe } \\
\hline Sim & 56 & 72,7 \\
\hline Não & 19 & 24,7 \\
\hline Não respondeu & 02 & 2,6 \\
\hline \multicolumn{3}{|c|}{$\begin{array}{l}\text { Equipe cirúrgica completa presente, presta atenção e participa } \\
\text { da checagem }\end{array}$} \\
\hline Sim & 27 & 35,1 \\
\hline Não & 50 & 64,9 \\
\hline \multicolumn{3}{|c|}{ Existência de itens da LVSC que não são checados } \\
\hline Sim & 19 & 24,7 \\
\hline Não & 57 & 74,0 \\
\hline Não respondeu & 01 & 1,3 \\
\hline
\end{tabular}

LVSC: lista de verificação de segurança cirúrgica. relacionados a duas etapas da LVSC, a saber: previsão de perda sanguínea (entrada) e contagem cirúrgica (saída) $(\mathrm{n}=1)$ e questões relacionadas aos equipamentos (pausa) e contagem cirúrgica (saída) $(\mathrm{n}=1)$; dois enfermeiros $(10,5 \%)$ não informaram os dados.

\section{DISCUSSÃO}

Neste estudo, os resultados indicaram o predomínio de dois formatos da LVSC disponíveis para uso em SO, a realização de programa educacional para a equipe de enfermagem e o circulante como responsável pela checagem da ferramenta na SO. Também em percentuais maiores, os resultados apontaram os enfermeiros como responsáveis pela iniciativa e pelo planejamento do processo de implementação da LVSC. Esses resultados foram corroborados com dados de pesquisa sobre como ocorreu o processo de implementação da LVSC em hospitais no Canadá ${ }^{10}$.

Para a implementação efetiva da LVSC, sugere-se que o processo seja conduzido por equipe multidisciplinar. Recomenda-se a inclusão de representantes de cada função (cirurgião, anestesista, enfermeiro, circulante e instrumentador) e pessoas com as seguintes características: respeitadas pelos pares, entusiasmadas, comprometidas e interessadas em iniciativas para melhorar a segurança do paciente e que acreditam que a comunicação e o trabalho em equipe possam ser otimizados. Especialmente, os cirurgiões e anestesistas, para serem recrutados, devem possuir disponibilidade, exercer boa influência e ter imagem positiva com seus pares.

Em investigação similar, o uso do formato impresso da LVSC (73\%) prevaleceu sobre os demais ${ }^{8}$. Contudo não há evidências sobre qual é o tipo de formato da lista que melhor favoreça o desempenho da equipe na checagem ${ }^{3,5}$.

A maioria dos enfermeiros indicou a realização de programa educacional, apenas com a participação da equipe de enfermagem, previamente à introdução da LVSC em SO, com conteúdo centrado nas explicações sobre por que e como utilizar a ferramenta. Ademais, 12 enfermeiros indicaram que essa estratégia não foi ofertada. Na literatura, é consensual que a educação, com envolvimento de todas as categorias profissionais, é elemento essencial e facilitador da implementação da LVSC ${ }^{3,5,11}$. Ante a diversidade de estratégias educativas utilizadas, o envolvimento de somente algumas categorias profissionais ${ }^{3,11}$ ou a ausência de processo educacional, a educação como facilitador pode tornar-se barreira ${ }^{12}$. 
Para melhor subsidiar esse elemento fundamental e insubstituível, recomenda-se a educação como um processo mais abrangente sob a tríade:

- conversa informal com cada membro da equipe cirúrgica: o diálogo visa conectar cada profissional com a ideia e o propósito da LVSC e, diretamente, solicitar colaboração para uso da ferramenta, antes da efetiva introdução em SO;

- treinar cada membro da equipe cirúrgica antes do uso efetivo: a abordagem inclui explicação de como fazer, demonstrar e dar oportunidade para a equipe cirúrgica praticar exaustivamente a checagem. Para viabilidade dessa etapa, pode-se treinar membros da equipe individualmente, em grupo ou a equipe cirúrgica completa ${ }^{5}$;

- treinamento continuado e orientação in loco, pela introdução da LVSC na SO: os membros da equipe precisam ser apoiados e orientados para melhorar o desempenho no uso diário ${ }^{5,11}$.

No que se refere ao responsável pela condução da checagem diária da LVSC na SO, os percentuais maiores indicados pelos participantes foram o circulante de sala, seguido pelo enfermeiro do CC. Em outros estudos, os resultados evidenciaram que o circulante coordenou o processo de checagem da LVSC $^{8}$ ou que a responsabilidade pela checagem da ferramenta foi compartilhada entre as diferentes categorias profissionais ${ }^{12,13}$.

Com base na análise das evidências disponíveis, elaborou-se um guia para implementação mais assertiva e sustentabilidade da LVSC. Em linhas gerais, os seguintes passos são necessários: a composição de equipe multidisciplinar para planejar e executar a implementação, com definição de papéis, expectativas e processos; a equipe de implementação deve conhecer os aspectos históricos e os objetivos relativos ao checklist; a avaliação do ambiente de trabalho com observação in loco da atuação das equipes e da dinâmica do grupo no contexto da SO e avaliação da cultura; a tomada de decisão para introduzir a LVSC, ou seja, decidir se o momento é oportuno para introduzir a ferramenta com base nas avaliações preliminares mencionadas; a adaptação e o teste da LVSC, considerada uma das tarefas-chave da equipe de implementação, anterior ao uso da ferramenta; o planejamento, que inclui definir o que a equipe de implementação vai fazer, como e quando, para disseminar o uso da ferramenta com lista das tarefas relacionadas a cada ação e elaboração de cronograma. Nessa fase, devem-se realizar conversas individuais, promover o uso da ferramenta, a educação/formação para a equipe, o treinamento, a supervisão in $l o c 0^{5}$.

A realização de conversas particulares é considerada a primeira etapa de aprendizagem da equipe cirúrgica. Destaca-se que não há substituição para essa conversa com cada membro da equipe. Nesse momento da conversa, é recomendável promover a LVSC por meio de estratégias criativas, transmitir mensagens sobre a ferramenta, a apresentação dos esforços empreendidos e os progressos obtidos até o momento. A segunda etapa de aprendizagem consiste em treinar e disseminar, o objetivo é explicar e demonstrar como deve ser utilizada e dar oportunidade para a equipe praticar. $\mathrm{O}$ acompanhamento, o feedback, o apoio e o treinamento continuado in loco são considerados como a terceira parte do processo de aprendizagem para uso efetivo da LVSC e sustentabilidade ao longo do tempo. Esse passo é realizado por um coach, membro da equipe recrutado e previamente capacitado para essa função, com vistas à melhoria do desempenho cotidiano da equipe; promover melhoria contínua por meio de revisões periódicas pode ajudar na utilização adequada da lista e auxiliar as equipes na adaptação às novas condições ${ }^{5}$.

Na presente pesquisa, em relação ao uso diário da LVSC, os resultados evidenciaram que a maioria dos enfermeiros respondeu que a equipe cirúrgica adere parcialmente ao uso da ferramenta. A adesão é diferente entre as etapas de checagem, com maior adesão na entrada e menor na saída. Entre as categorias profissionais, houve adesão maior pela equipe de enfermagem e menor pelos cirurgiões e, ainda, a existência de itens da LVSC que não são checados; itens da pausa e saída foram os mais negligenciados.

Em estudo nacional, os autores analisaram 375 prontuários médicos de pacientes cirúrgicos. Os resultados demonstraram adesão ao uso da LVSC de $60 \%$, mas apenas em $4 \%$ o impresso estava completamente preenchido ${ }^{8}$. Ressalta-se que, nos locais onde as taxas de adesão à LVSC são elevadas, muitas vezes, a qualidade do preenchimento e a fidedignidade aos objetivos da ferramenta estão comprometidas ${ }^{11}$.

No presente estudo, a maioria dos enfermeiros respondeu que houve incremento da adesão desde o início da implementação da LVSC nos serviços de saúde pesquisados. Contudo, para 33,8\% dos participantes, não houve aumento da adesão pela equipe cirúrgica. Outros estudos evidenciaram resultados semelhantes, uma vez que o uso do checklist não se sustentou ao longo do tempo ${ }^{13,14}$.

Com relação à diferença na adesão entre as etapas da LVSC, os dados do presente estudo foram semelhantes àqueles identificados em pesquisa nacional realizada em três hospitais do 
Distrito Federal. A adesão à etapa de saída foi inferior às duas primeiras etapas, principalmente em relação ao item relacionado aos problemas com equipamentos e à contagem cirúrgica. Nos casos em que a contagem cirúrgica foi executada, o procedimento ocorreu após a saída do paciente da $\mathrm{SO}^{8}$.

Uma explicação possível para a adesão baixa à última etapa da LVSC pode pela saída de membros da equipe cirúrgica da $\mathrm{SO}$ antes do término do procedimento ${ }^{15}$.

Quando uma etapa da LVSC é omitida, sem ocorrer danos para o paciente, o uso inadequado é facilmente incorporado pela equipe; nessas circunstâncias, essa ferramenta pode ser considerada uma barreira de segurança fraca ${ }^{16}$.

Na literatura, há evidências que comprovam a resistência diferente na utilização da LVSC entre as categorias profissionais, o apoio ao uso da ferramenta tende a ser maior pelos enfermeiros e anestesistas do que pelos cirurgiões ${ }^{3,4}$. Geralmente, os enfermeiros estão mais habituados com o uso de checklists como um componente estruturado de atendimento clínico, o que pode favorecer a adesão dessa categoria ${ }^{17}$. Em contrapartida, os médicos acreditam que o uso formal da LVSC é redundante, uma vez que já promulgam os princípios de segurança na prática clínica. Portanto o uso sustentado da LVSC pode ser específico de cada disciplina e bem-sucedido quando os médicos são ativamente engajados no processo ${ }^{4}$.

Neste estudo, a maioria dos enfermeiros respondeu que, na checagem diária da LVSC, a equipe cirúrgica completa não está presente, não presta atenção e não ocorre participação ativa de membros da equipe. Ante essas inadequações, pode-se inferir que, nesses locais, talvez o uso da LVSC foi compreendido como mero exercício de assinalar itens, e não como prática que favoreça a prevenção de eventos adversos, a melhoria da comunicação e o trabalho em equipe, ou seja, compreensão contraditória ao que é preconizado pela OMS.

Em estudo desenvolvido em cinco hospitais da Inglaterra com foco na observação da execução da LVSC nas SO, os resultados foram semelhantes. Em $40 \%$ dos casos, os membros da equipe estavam ausentes na pausa e na saída e não conseguiram interromper outras atividades em aproximadamente $70 \%$ dos casos. O desempenho mais adequado ocorreu quando os cirurgiões conduziram a checagem: todos os membros estavam presentes e interromperam as demais atividades para cumprir os itens da LVSC ${ }^{15}$.

No serviço de saúde, o fato de a LVSC não ser executada de forma fidedigna à recomendação da OMS não deve ser encarado com desânimo, pois revela possibilidade para aprimoramento do processo de implementação ${ }^{18}$. Em geral, a implementação de intervenções comportamentais concebidas para qualificar a prática clínica é permeada com nuances de sucesso ou fracasso, pois raramente a experiência é bem-sucedida ou sem sucesso em sua totalidade. A implementação da LVSC pode promover a qualificação do processo de trabalho e do trabalho em equipe em alguns contextos, mas falha ou tem sucesso limitado em outros. Considerar tais premissas na implementação dessa ferramenta é fundamental para elencar abordagens mais compatíveis com a realidade institucional ${ }^{4}$.

A checagem da LVSC não deve ser limitada à confirmação da identidade do paciente, da operação e do sítio cirúrgico, dos instrumentos necessários, dos fluidos, dos hemoderivados e dos equipamentos disponíveis. Também deve incluir a apresentação de todos os membros da equipe, o cirurgião deve informar sobre as etapas críticas da cirurgia e resolver quaisquer preocupações verbalizadas pela equipe de anestesia e de enfermagem ${ }^{19}$. A falta de participação ativa de membros da equipe infringe os princípios norteadores da LVSC, uma vez que o diálogo pode melhorar o cuidado cirúrgico e mudar positivamente a forma como os membros da equipe cirúrgica interagem uns com os outros e com os pacientes. Portanto esses itens não devem ser removidos ou negligenciados ${ }^{5}$.

Em estudo norte-americano, conduzido em 2018, os autores descreveram a experiência de hospitais na implementação da LVSC de 2010 a 2017. Desse processo, apresentaram-se três lições para formuladores de políticas de hospitais em nível local, estadual ou nacional:

- programa bem-sucedido deve ser planejado para envolver todas as partes interessadas (médicos, enfermeiros, técnicos de enfermagem, instrumentadores, entre outros);

- oferecimento de uma variedade de estratégias (processo educativo que inclua reuniões presenciais, seminários online, treinamento presencial in loco e visita de acompanhamento);

- processo de implementação que proponha mudanças no processo convencional precisará de tempo e recursos ${ }^{20}$.

No contexto da cirurgia segura, acredita-se que a liderança participativa da enfermagem, por meio da comunicação entre a equipe com o paciente, com os familiares e com os gestores hospitalares, contribuirá para promover o cuidado centrado no paciente, de forma continuada e segura ${ }^{21}$.

Com relação às limitações, recomenda-se cautela na generalização dos resultados indicados, uma vez que o estudo foi conduzido em dois municípios do estado do Paraná. A análise 
dos dados pautou-se nas informações relatadas pelos enfermeiros atuantes em CC. Assim, aspectos relevantes sobre o processo de implementação da LVSC podem ter sido referidos de maneira diferente da realidade. Por exemplo, o relato de dados sobre a adesão e os demais aspectos de uso da LVSC podem ter sido mais positivos do que, efetivamente, ocorre na prática, quando o contexto é observado diretamente.

\section{CONCLUSÃO}

Com relação às principais conclusões do estudo, evidenciou-se que a LVSC foi disponibilizada em dois formatos para uso em SO; o programa educacional foi estratégia relevante realizada, mas oferecido predominantemente para a equipe de enfermagem, e a checagem da ferramenta, na maioria das vezes, envolveu apenas o circulante de sala. Os enfermeiros foram os principais responsáveis pela iniciativa e pelo planejamento do processo de implementação do checklist em questão.
$\mathrm{Na}$ opinião da maioria dos enfermeiros, a adesão ao uso da ferramenta é parcial, com diferenças entre as etapas de checagem, sendo maior na entrada e menor na saída. A adesão ao uso diário da LVSC é maior pela equipe de enfermagem e menor pelos cirurgiões. E, entre as etapas de checagem, itens da pausa e saída foram os mais negligenciados.

No que se refere às implicações para a enfermagem perioperatória, as evidências geradas oferecem subsídios para o conhecimento de como ocorreu a implementação da LVSC, seu uso diário e quais estratégias foram adotadas nesse processo, no contexto brasileiro. Portanto tais evidências contribuem para reduzir as lacunas de conhecimento e promover o avanço científico dessa área da enfermagem. Além disso, o conhecimento produzido auxilia a implementação da ferramenta em serviços que ainda não utilizam tal prática, bem como a revisão naqueles que já adotaram a ferramenta no seu cotidiano. Em suma, as evidências geradas acarretam incremento na qualidade da assistência prestada e promoção permanente da segurança do paciente cirúrgico.

\section{REFERÊNCIAS}

1. Weiser TG, Haynes AB. Ten years of the surgical safety checklist. $\mathrm{Br}$ J Surg. 2018;105(8):927-9. https://doi.org/10.1002/bjs.10907

2. World Health Organization. WHO guidelines for safe surgery 2009. Safe Surgery Saves Lives [Internet]. Genebra (CHE): WHO; 2009 [acesso em 18 set. 2017]. 123p. Disponivel em: https://www.who. int/patientsafety/safesurgery/tools_resources/9789241598552/en/

3. Treadwell JR, Lucas S, Tsou AY. Surgical checklists: a systematic review of impacts and implementation. BMJ Qual Saf. [Internet]. 2014 [acesso em 6 maio 2020]; 23(4):299-318. Disponível em: https://pubmed.ncbi. nlm.nih.gov/23922403/https://doi.org/10.1136/bmjqs-2012-001797

4. Gillespie BM, Marshall A. Implementation of safety checklists in surgery: a realist synthesis of evidence. Implement Sci [Internet]. 2015 [acesso em 11 maio 2020]; 10:137. Disponível em: https://pubmed.ncbi.nlm. nih.gov/26415946/ https://doi.org/10.1186/s13012-015-0319-9

5. Ariadne Labs. Surgery: WHO Safe Surgery Checklist Resources [Internet]. Boston: Ariadne Labs; 2015 [acesso em 23 fev. 2017]. Disponível em: https://www.ariadnelabs.org/areas-of-work/ safe-surgery-checklist/resources/\#Downloads\&\%20Tools

6. Tostes MFP, Galvão CM. Processo de implementação da Lista de Verificação de Segurança Cirúrgica: revisão integrativa. Rev Latino-Am Enfermagem [Internet]. 2019 [acesso em 3 maio 2020];27:e3104. Disponível em: https://doi.org/10.1590/1518-8345.2921.3104

7. Freitas MR, Antunes AG, Lopes BNA, Fernandes FC, Monte LC, Gama ZAS. Avaliação da adesão ao checklist de cirurgia segura da OMS em cirurgias urológicas e ginecológicas, em dois hospitais de ensino de Natal, Rio Grande do Norte, Brasil. Cad Saúde Pública [Internet]. 2014 [acesso em 3 maio 2020];30(1):137-48. Disponivel em: https:// doi.org/10.1590/0102-311X00184612

8. Santana HT, Freitas MR, Ferraz EM, Evangelista MS. WHO safety surgical checklist implementation evaluation in public hospitals in the Brazilian Federal District. J Infect Public Health [Internet]. 2016 [acesso em 3 maio 2020];9(5):586-99. Disponivel em: https://pubmed.ncbi.nlm.nih. gov/26924253/. https://doi.org/10.1016/j.jiph.2015.12.019

9. Cadman V. Use of the WHO Surgical Safety Checklist in low and middle income countries: areview of the literature. J Perioper Pract. [Internet]. 2018 [acesso em 3 maio 2020];28(12):334-8. Disponível em: https://doi.org/10.1177\%2F1750458918776551

10. Gagliardi AR, Straus SE, Shojania KG, Urbac DR. Multiple interacting factors influence adherence, and outcomes associated with surgical safety checklists: a qualitative study. PloS One [Internet]. 2014 [acesso em 12 maio 2020];9(9):e108585. Disponível em: https:// pubmed.ncbi.nlm.nih.gov/25260030/. https://doi.org/10.1371/ journal.pone. 0108585

11. Nugent E, Hseino H, Ryan K, Traynor O, Neary P, Keane FBV. The surgical safety checklist survey: a national perspective on patient safety. Ir J Med Sci [Internet]. 2013 [acesso em 12 maio 2020]; 182(2):1716. Disponível em: https://doi.org/10.1007/s11845-012-0851-4

12. O'Connor P, Reddin C, O'Sullivan M, O'Duffy F, Keogh I. Surgical checklists: the human factor. Patient Saf Surg [Internet]. 2013 [acesso em 12 maio 2020];7(1):14-20. Disponível em: https://doi. org/10.1186/1754-9493-7-14 
13. Ong APC, Devcich DA, Hannam J, Lee T, Merry AF, Mitchell SJ. A 'paperless' wall-mounted surgical safety checklist with migrated leadership can improve compliance and team engagement. BMJ Qual Saf [Internet]. 2016 [acesso em 12 maio 2020];25(12):971-6. Disponível em: https://doi.org/10.1136/bmjqs-2015-004545

14. Lilaonitkul M, Kwikiriza A, Ttendo S, Kiwanuka J, Munyarungero E, Walker IA, et al. Implementation of the WHO surgical safety checklist and surgical swab and instrument counts at a regional referral hospital in Uganda-a quality improvement project. Anaesthesia [Internet]. 2015 [acesso em 12 maio 2020];70(12):1345-55. Disponível em: https://doi.org/10.1111/anae.13226

15. Russ S, Rout S, Caris J, Mansell J, Davies R, Mayer E, et al. Measuring variation in use of the WHO Surgical Safety Checklist in the operating room: a multicenter prospective cross-sectional study. J Am Coll Surg [Internet]. 2015 [acesso em 12 maio 2020];220(1):1-11.e4. Disponível em: https://doi.org/10.1016/j. jamcollsurg.2014.09.021

16. Rydenfält C, Ek A, Larsson PA. Safety checklist compliance and a false sense of safety: new directions for research. BMJ Qual Saf [Internet]. 2014 [acesso em 12 maio 2020];23(3):183-6. Disponível em: https://doi.org/10.1136/bmjqs-2013-002168
17. Weiser TG, Berry WR. Review article: perioperative checklist methodologies. Can J Anaesth [Internet]. 2013 [acesso em 12 maio 2020];60(2):136-42. Disponível em: https://doi.org/10.1007/ s12630-012-9854-x

18. Rydenfält C, Johansson G, Odenrick P, Akerman K, Larsson PA. Compliance with the WHO surgical safety checklist: deviations and possible. Int J Qual Health Care [Internet]. 2013 [acesso em 12 maio 2020];25(2):182-7. Disponível em: https://doi.org/10.1093/intqhc/mzt004

19. Leape LL. The Checklist Conundrum. N Engl J Med [Internet]. 2014 [acesso em 12 maio 2020];370(11):1063-4. Disponível em: https:// doi.org/10.1056/nejme1315851

20. Berry WR, Edmondson L, Gibbons LR, Childers AK, Haynes AB, Foster R, et al. Scaling safety: the south Carolina Surgical Safety Checklist experience. Health Affairs [Internet]. 2018 [acesso em 123 maio 2020];37(11):1779-86. Disponível em: https://doi.org/10.1377/ hlthaff.2018.0717

21. Oliveira MCB, Korb A, Zocche DAA, Cabral DB, Pertille F, Frigo J. Adesão do checklist cirúrgico à luz dacultura de segurança do paciente. Rev SOBECC [Internet]. 2018 [acesso em 123 maio 2020];23(1):36-42. Disponível em: https://doi.org/10.5327/Z1414-4425201800010007 